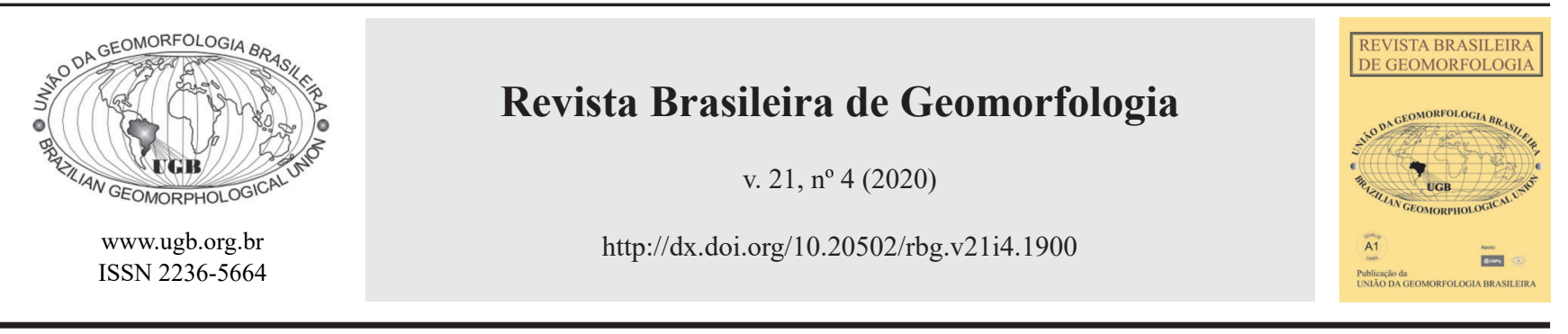

\title{
TRANSPORTE DE SEDIMENTOS INDUZIDO POR ONDAS NO SEGMENTO NORTE DA ENSEADA DA BAIA FORMOSA, RIO DAS OSTRAS, ESTADO DO RIO DE JANEIRO
}

\author{
WAVE-INDUCED SEDIMENT TRANSPORT ALONG THE NORTHERN \\ STRETCH OF ENSEADA DA BAIA FORMOSA - RIO DE JANEIRO STATE
}

\author{
Daniel Fernandes \\ Museu Nacional, Universidade Federal do Rio de Janeiro \\ Quinta da Boa Vista, s/n, Rio de Janeiro, Rio de Janeiro. CEP: 20940-040. Brasil \\ ORCID: http://orcid.org/0000-0003-4218-8198 \\ E-mail:fernandes.geol@gmail.com
}

João Wagner de Alencar Castro

Museu Nacional, Universidade Federal do Rio de Janeiro

Quinta da Boa Vista, s/n, Rio de Janeiro, Rio de Janeiro. CEP: 20940-040. Brasil ORCID: http://orcid.org/0000-0002-8955-5971

E-mail:jwacastro@gmail.com

\begin{tabular}{l} 
Informações sobre o Artigo \\
\hline Recebido (Received): \\
15/04/2020 \\
Aceito (Accepted): \\
06/07/2020
\end{tabular}

\section{Palavras-chave:}

Ondas; Transporte longitudinal de sedimentos; Enseada da Baia Formosa - Rio de Janeiro

\section{Keywords:}

Waves; Longitudinal sediment transport; Enseada da Baia Formosa - Rio de Janeiro State

\begin{abstract}
Resumo:
As mudanças morfológicas em ambientes costeiros são produtos de interações entre o oceano e o continente, onde as ondas são o principal agente responsável pelo "input" de energia no transporte de sedimentos. O presente manuscrito tem como objetivo quantificar taxas de transporte de sedimentos induzidos por ondas nas praias da Tartaruga e Abricó, segmento norte da Enseada da Baia Formosa, Rio das Ostras - Estado do Rio de Janeiro. Como procedimento metodológico realizouse levantamento histórico do clima de ondas e tratamento estatístico de dezesseis amostras de sedimentos coletados na zona de surfe (antepraia) em diferentes datas. Os principais parâmetros sedimentológicos utilizados foram o diâmetro mediano do grão $\left(\mathrm{D}_{50}\right)$, grau de seleção $(\sigma)$ e grau de assimetria $(S k)$. As estimativas de taxas de transporte de sedimentos foram obtidas através da expressão matemática proposta por KOMAR \& INMAN (1970) também conhecida como fórmula do CERC (1984). Resultados obtidos sugerem predomínio de transporte residual de norte para sul, com taxas entre $75.000 \mathrm{~m}^{3} . \mathrm{ano}^{-1}$ a $515.000 \mathrm{~m}^{3}$.ano $\mathrm{o}^{-1}$. A disparidade entre os valores obtidos associa-se a frequência e alturas de ondas significativas em águas profundas $\left(H_{0}\right)$ para os diferentes quadrantes e também às variações texturais dos sedimentos analisados.
\end{abstract}

\section{Abstract:}

The morphological changes in coastal environments are the product of interactions between the oceanographic and continental processes, where the waves are the 
main responsible agent for the energy input for sediment transport. The present manuscript aims to estimate waveinduced sediment transport rate on Tartaruga and Abricó beaches, northern segment of the Enseada da Baia Formosa, Rio das Ostras - Rio de Janeiro State. As a basic methodology, a historical survey of oceanic data was performed and a statistical treatment was performed with 16 sediment samples collected in the surf zone at different dates. The main statistical parameters were the grain size $\left(\mathrm{D}_{50}\right)$, mean cube deviation $(\sigma)$ and skewness $(S k)$. The sediment transport's rate was obtained using The KOMAR and INMAN (1970) or so-called CERC (1984) equation. In general, the results represent the predominance of residual transport from north to south with rates between 75,000 $\mathrm{m}^{3}$.year-1 to $515,000 \mathrm{~m}^{3}$.year-1. The disparity between the values obtained is related to the frequency, the significant wave heights in deep waters $\left(H_{0}\right)$ for the different analyzed quadrants and the textural variations of the sediments.

\section{Introdução}

As zonas costeiras são reconhecidas do ponto de vista geomorfológico, como áreas extremamente sensíveis e dinâmicas (Castro et al., 2011). A manutenção do perfil morfológico ao longo do tempo no ambiente praial não significa repouso dos sedimentos, mas, equilíbrio entre entrada e saída de sedimentos na zona costeira (Souza, 2009). Em consonância, os estudos relacionados ao transporte de sedimentos induzido por ondas, especialmente o transporte longitudinal, tem sido foco de pesquisadores e profissionais das áreas de geociências e engenharia costeira. Compreender o mecanismo de transporte sedimentar na zona de surfe (antepraia) é fundamental para a determinação de parâmetros utilizados na gestão de obras públicas e privadas nas zonas costeiras.

A dinâmica das praias é ocasionada pela atuação de processos oceanográficos, entre estes, ondas, correntes e marés, sendo as ondas, responsáveis pelo maior "input" de energia no ambiente praial (Dean \& Dalrymple, 2002). Ao se aproximarem da antepraia, em ambiente de águas rasas $(\mathrm{H} / \mathrm{L}<0,05)$, devido aos efeitos da morfologia do fundo plataformal, as ondas começam a ser refratadas (Silva et al., 2004; Castro et al., 2011). A refração é responsável pelo alinhamento da zona de arrebentação das ondas, exercendo um paralelismo em relação à praia, gerando assim, um vetor resultante entre a senóide e o substrato (Suguio, 2003). Esse processo hidrodinâmico denomina-se de deriva litorânea, um dos principais agentes do transporte de sedimentos.

No ambiente praial, o ataque obliquo das ondas é proveniente de diversos quadrantes, produzindo dia-a-dia ou sazonalmente uma inversão da direção do transporte longitudinal de sedimentos (CERC,1984). Assim, as taxas de transporte longitudinal podem ser positivas ou negativas de acordo com a convenção adotada para sentido das correntes de deriva litorânea. Os volumes de materiais transportados variam de zero a milhões de metros cúbicos de sedimento ao longo do ano.

Desde os anos sessenta do século passado diversos pesquisadores vêm desenvolvendo estudos, envolvendo fórmulas e metodologias que resultem em estimativas precisas e confiáveis do transporte de sedimentos induzido por ondas. Ano após ano, observa-se o surgimento de novas fórmulas e métodos para o cálculo do transporte de sedimentos litorâneo (Alfredine, 1999). Tal fato, ocorre principalmente devido ao avanço dos recursos computacionais e também ao aumento da disponibilidade de dados, relacionados a medições e monitoramento de praias em todo o mundo.

As taxas de transporte longitudinal de sedimentos são mensuradas em função de variáveis hidrodinâmicas e sedimentológicas por fluxo de energia (Longuet-Hingins \& Stewart, 1964; Bijker, 1967; Komar, 1976; Bilard \& Inman, 1981; Van Rijn, 1984; CERC, 1984; Bayran et al., 2001; Haas \& Haynes, 2004). Muitos desses pesquisadores propõem expressões baseadas em análises dimensionais ajustadas a partir de dados de experimentos realizados em laboratório. Este artigo objetiva avaliar qualitativamente e quantitativamente as taxas de transporte de sedimentos induzidos por ondas no segmento norte do arco praial da Baia Formosa, Rio das Ostras / RJ, a partir da formulação matemática proposta pelo CERC (1984). Nessa formulação, utiliza-se o coeficiente adimensional $K$ correspondente ao diâmetro mediano do grão $\left(\mathrm{D}_{50}\right)$ e $P$, a potência induzida pelas ondas na zona de arrebentação correspondente a ante praia.

\section{Contexto Ambiental da Área de Estudo}

Trabalhos desenvolvidos por Castro et al. (2014) sugerem que na transição entre o Pleistoceno e o Holoceno, há 11.500 anos A.P, toda plataforma continental interna da região de estudo encontrava-se exposta. Após o optimum climático holocênico, o nível relativo do mar 
atingiu 3,0 $\mathrm{m}$ acima do atual entre 4590 - 5100 anos cal A.P (op. cit.). Durante esse evento transgressivo, o segmento litorâneo aqui estudado caracterizava-se por reentrâncias, baias e deltas intralagunares. À medida que o nível relativo do mar foi baixando, desenvolveu-se corpos lagunares e extensos cordões litorâneos regressivos. Paulatinamente, com o desenvolvimento da planície costeira, estabeleceram-se os pântanos costeiros e os rios Una e São João.

Aárea de estudo, localiza-se no segmento norte da enseada da Baia Formosa, entre a Praia das Tartarugas e Praia do Abricó, município de Rio das Ostras - Estado do Rio de Janeiro (Figura 1). Essa região vem passando por intenso processo erosivo há aproximadamente duas décadas, resultando na transgressão da linha de praia e consecutivas intervenções com intuito de mitigar os impactos na zona costeira. O processo de erosão costeira acarretou na destruição de parte da infraestrutura urbana e propriedades privadas na cidade de Rio das Ostras (Castro et al., 2011).

Do ponto de vista morfológico, a enseada da Baia Formosa caracteriza-se por um grande arco praial de orientação preferencial norte-sul com aproximadamente $26 \mathrm{~km}$ de extensão, limitado pelo Cabo Búzios e pelo promontório de Rio das Ostras. Subdivide-se em dois segmentos bem distintos. No segmento sul, área do Cabo de Búzios, as praias caracterizam-se por estado morfodinâmico dissipativo, conforme modelo proposto por Wright \& Short (1984). A zona de surfe é larga com baixo gradiente topográfico. Os sedimentos são predominantemente de finos a médios. A antepraia caracteriza-se por elevado estoque de areia, denotando feições em barras paralelas (Muehe et al., 2011). No extremo oposto, segmento norte, as praias apresentam estado morfodinâmico de intermediário a refletivo. As estruturas sedimentares resultantes caracterizam-se por megacúspides e fortes correntes de retorno (Short \& Hogan, 1994.). Nesse segmento, município de Rio das Ostras, registra-se a presença de sedimentos predominantemente grossos, tanto na zona de surfe (antepraia), como na face da praia (Oliveira \& Muehe, 2013).

Na plataforma continental interna da enseada da Baia Formosa, os sedimentos apresentam-se predominantemente grossos e bem selecionados na faixa mais próxima a linha de costa. Verificou-se tendência de aumento do desvio padrão em direção à faixa de sedimentos finos, entre as isóbatas de 25 a 45 m (Fernandez
\& Muehe, 1995). Na plataforma continental externa, prevalece a ocorrência de sedimentos tipicamente carbonáticos provenientes de algas calcáreas nodulares (Alves \& Ponzi, 1984; Carvalho \& Rizzo, 1994).

Trabalhos desenvolvidos na Bacia de Campos sugerem que o clima de ondas é predominantemente de tempo bom, com ondulações provenientes do quadrante nordeste. Registra-se também a ocorrência de marulhos "sweell" provenientes do Anticiclones do Atlântico Sul, associados aos sistemas frontais e frentes frias polares, com direção leste-sudeste (Pinho, 2003; Savi, 2007; Castro et al., 2011; Parente et al., 2014). Conforme informações da Marinha do Brasil referentes aos ondógráfos instalados na Bacia de Campos, verifica-se a ocorrência de frequentes trens de ondas de nordeste e sul-sudeste com alturas em águas profundas $\left(H_{0}\right)$ de 0,5 a 6,0 m e períodos $(T)$ de 2,0 a $17,0 \mathrm{~s}$.

\section{Materiais e Métodos}

Foi realizado o levantamento histórico do clima de ondas, regime de marés e tratamento estatístico de amostras de sedimentos coletados na zona de surfe (antepraia) em diferentes períodos. Nas análises de sedimentos, utilizou-se os parâmetros sedimentológicos referentes ao diâmetro mediano do grão $\left(\mathrm{D}_{50}\right)$, grau de seleção $(\sigma)$, grau de assimetria $(S k)$ e curtose. Além disso, a expressão matemática proposta por KOMAR \& INMAN (1970), também conhecida como fórmula do CERC (USACE, 1984; USACE, 2002a, b), foi aplicada para estimar as taxas de transporte de sedimentos induzidas por ondas.

\subsection{Análises granulométricas}

As amostras utilizadas fazem parte do acervo do Laboratório de Geologia Costeira, Sedimentologia e Meio Ambiente - LAGECOST, do Museu Nacional / UFRJ. Foram obtidas através de campanhas realizadas durante os anos de 2009 e 2010 , para desenvolvimento do projeto Variações do nível relativo do mar e reconstrução paleoambiental durante o Holoceno na planície costeira do Rio de Janeiro, Sudeste Brasileiro, que conta com apoio do Conselho Nacional de Desenvolvimento Científico e Tecnológico e Fundação Carlos Chagas Filho de Amparo à Pesquisa do Estado do Rio de Janeiro - FAPERJ 

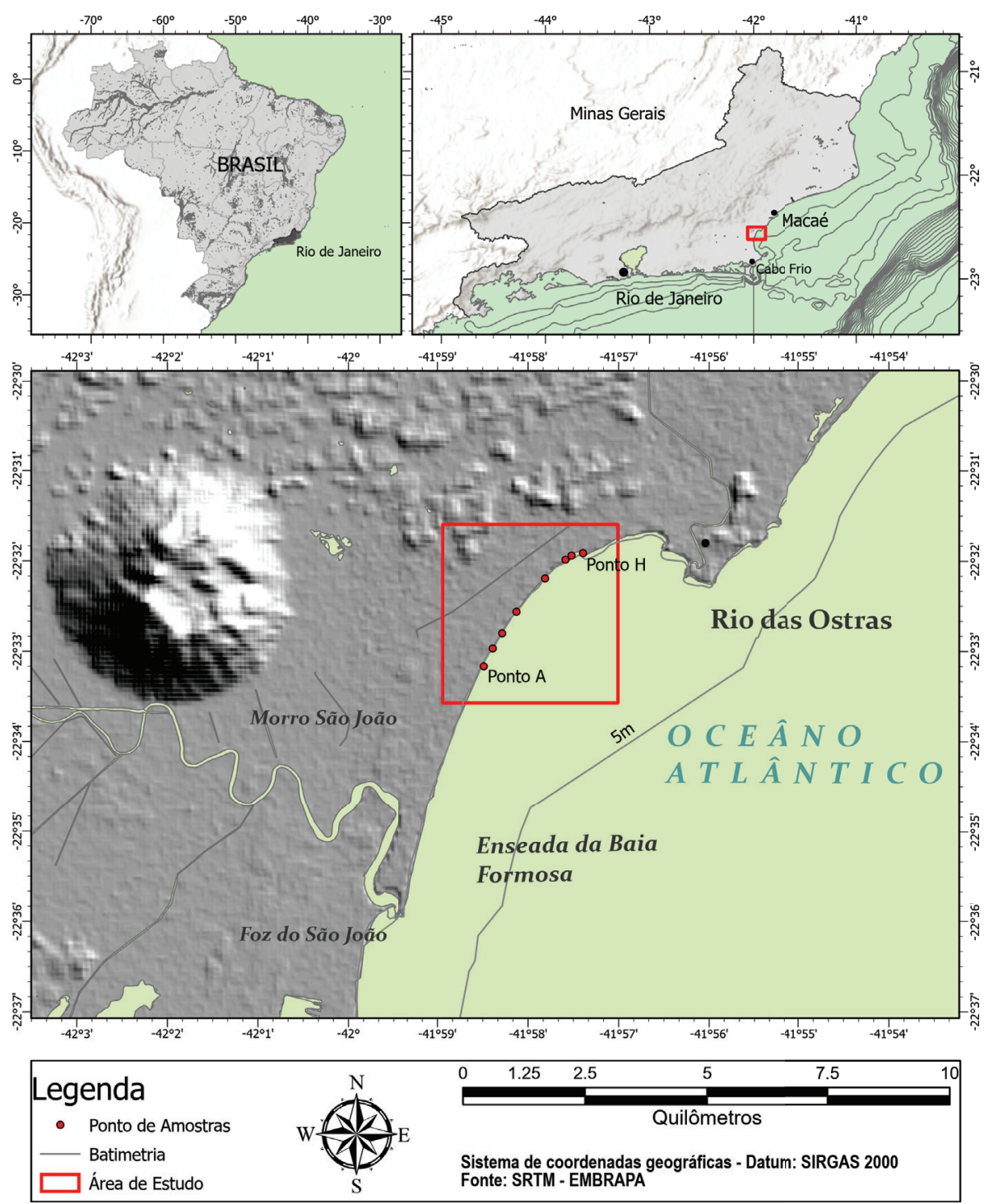

Figura 1 - Localização da área de estudo no contexto da Região dos lagos Fluminenses - Rio de Janeiro. O polígono em vermelho representa a região da praia das Tartarugas e Abricó, segmento em processo intenso de erosão costeira. As áreas de coleta de sedimentos estão representadas por pontos vermelhos no mapa, sendo o ponto localizados mais a sul indicado pela letra " $A$ " e mais ao norte pela letra " $H$ ".

Inicialmente, foram estabelecidos 8 (oito) pontos para controle e coleta de sedimentos na zona de surfe, denominados pelas letras "A" à “H”, indicados na figura 1. Em cada um destes pontos foram coletadas duas amostras para períodos distintos. Foi definido o subscrito (v) para amostras coletadas no período de verão de 2010 e (i) para amostras coletadas durante inverno de 2009. Através destas amostras, foram definidos o $\mathrm{D}_{50}, \sigma, S k$ e curtose para cada amostra coletada (Friedman, 1962b). Ressalta-se que durante a análise destas amostras, o material carbonático não foi retirado em função de sua importância para a composição do material presente em praias localizadas nos trópicos (Komar, 1976).

\subsection{Ondas}

Informações sobre as ondas na região foram obtidas junto ao Banco de Dados Oceanográficos - BNDO da Marinha do Brasil, referentes a diversos ondógrafos instalados na Bacia de Campos. Através da análise estatística da série histórica entre 1963 a 1999, foram estabelecidas direções, períodos $(T)$ e alturas de ondas em águas profundas $\left(H_{0}\right)$ necessários para definição do clima de ondas e estimativa da taxa de transporte de sedimentos longitudinal. 


\subsection{Estimativas de taxas de transporte de sedimentos}

A partir das informações sobre o clima de ondas da região, referentes aos períodos de inverno e verão, foram definidos os parâmetros hidrodinâmicos referentes à altura das ondas na arrebentação $\left(H_{b}\right)$ e profundidade de arrebentação $(h b)$, para ambos períodos. A equação 1 , que relaciona $H_{b}$ à $H_{0}$, é apresentada por Weishar \& Byrne (1978), e $h b$ é adaptada da teoria linear das ondas, conforme equação 2 .

$$
\begin{gathered}
H_{b}=0,39 g^{0,2}\left(\mathrm{TH}_{0}\right)^{0,4} \\
h b=\frac{H_{b}}{0,78}
\end{gathered}
$$

onde: $g$ é aceleração da gravidade, igual a 9,78 m.s ${ }^{-2}$

A estimativa de taxa de transporte longitudinal de sedimentos $(Q s)$ foi calculada pela variação do volume de sedimentos, expressa em $\mathrm{m}^{3} . \mathrm{s}^{-1}$, conforme equação 3 , proposta por CERC (USACE,1984). A fórmula é expressa como um método que tem como base o fluxo de energia.

$$
Q s=\frac{I}{(\rho s-\rho w) g(1-n)}
$$

sendo $\rho \boldsymbol{s}$ a densidade do material transportado $(2,65$ g. $\left.\mathrm{cm}^{-3}\right), \rho \boldsymbol{w}$ é a densidade da água $\left(1023 \mathrm{~kg} . \mathrm{m}^{-3}\right), \boldsymbol{n}$ representa a razão entre o volume de sólidos e o volume total, estimada em 0,35 (Dean, 2000), e I representando o peso imerso de sedimentos, associado empiricamente a potência induzida pelas ondas $(P)$ conforme equação 4 .

$$
I=K P
$$

onde $\boldsymbol{K}$ é um coeficiente empírico adimensional de proporcionalidade (Komar,1988), definido em função do $\mathbf{D}_{50}$ e P é a potência induzida pelas ondas na zona de arrebentação. As equações que se seguem foram obtidas através da expressão proposta CERC (USACE, 1984):

$$
K=1,4 e^{\left(2,5 D_{50}\right)}
$$

$$
\begin{gathered}
P=E C n \cos \alpha_{b} \sin \alpha_{b} \\
E=\frac{1}{8}\left(\rho g H_{b}{ }^{2}\right) \\
\alpha_{b}=\alpha_{0}\left(\frac{C}{C_{0}}\right)
\end{gathered}
$$

sendo $\boldsymbol{E}$ definido como a energia da onda na zona de arrebentação, $\boldsymbol{H}_{\boldsymbol{b}}$ a altura de onda na arrebentação, $\alpha_{b}$ é o ângulo de ataque das ondas em águas rasas e $\alpha_{0} \mathrm{o}$ ângulo de ataque em águas profundas.

\section{Resultados}

\subsection{Análises granulométricas}

Resultados estatísticos provenientes da análise granulométrica associados à definição do clima de ondas local são fundamentais para as estimativas de taxas e sentidos de transporte de sedimentos induzido por ondas. Resultados referentes ao $\mathrm{D}_{50}$, grau de seleção $(\sigma)$, assimetria $(S k)$ e curtose estão apresentados na tabela 1 , assim como as respectivas coordenadas para cada ponto de coleta. Na figura 2, são apresentadas curvas de frequências para cada ponto de coleta de amostra para períodos de inverno e verão.

Os resultados estatísticos (tabela 1) indicam que o segmento norte da Enseada da Baia Formosa é composto, predominantemente, por areias grossas a muito grossas, moderadamente selecionadas, com tendência a diminuição da granulometria de sul para norte. Assim como os valores de $S k$, que variaram entre $+0,89$ e $-1,48$, de $\mathbf{A}$ para $\mathbf{H}$, conforme figura 1.

Os valores estatísticos da curtose apresentados na tabela 1, assim como os gráficos de distribuição granulométrica, apresentados na figura 2, sugerem que amostras coletadas durante o período de inverno apresentam maior dispersão de classes, comparados ao período de verão. Neste, formas leptocúrticas são observadas, exceto para os pontos $\mathbf{B}$ e $\mathbf{C}$, onde ocorrem formas platicúrticas e mesocúrticas, respectivamente. 
Fernandes D. \& Castro J. W. A.

Tabela 1: Resultados dos parâmetros estatísticos (D50, grau de seleção, grau de assimetria e curtose) e coordenadas em WGS 84 / UTM 24S

\begin{tabular}{|c|c|c|c|c|c|c|c|c|c|c|}
\hline \multirow{2}{*}{ Amostras } & \multirow{2}{*}{\multicolumn{2}{|c|}{ Coordenadas }} & \multicolumn{2}{|c|}{ D $_{50}$ (Phi) } & \multicolumn{2}{|c|}{$\sigma$} & \multicolumn{2}{|c|}{$S K$} & \multicolumn{2}{|c|}{ Curtose } \\
\hline & & & Verão & Inverno & Verão & Inverno & Verão & Inverno & Verão & Inverno \\
\hline$A_{v-i}$ & 194043,4 & 7502932,3 & $-0,47$ & $-0,31$ & 0,96 & 1,04 & 0,89 & 0,79 & 3.90 & 3.68 \\
\hline $\mathrm{B}_{\boldsymbol{v}-i}$ & 194209,4 & 7503307,5 & 0,52 & $-0,18$ & 1,43 & 1,24 & 0,08 & 0,74 & 1.86 & 2.72 \\
\hline$C_{v-i}$ & 194381,1 & 7503614,3 & 0,06 & 0,08 & 1,08 & 1,18 & 0,32 & 0,22 & 2.64 & 2.34 \\
\hline$D_{v-i}$ & 194643,2 & 7504065,5 & 0,83 & 0,00 & 0,78 & 1,10 & 0,37 & 0,24 & 3.77 & 2.42 \\
\hline$E_{v-i}$ & 195170,6 & 7504760,5 & 0,74 & 0,63 & 0,96 & 1,08 & 0,20 & 0,42 & 3.17 & 2.61 \\
\hline$F_{v-i}$ & 195549,6 & 7505147,2 & 0,36 & 0,55 & 1,01 & 1,17 & $-0,09$ & $-0,06$ & 3.88 & 2.51 \\
\hline $\mathrm{G}_{\boldsymbol{v}-\boldsymbol{i}}$ & 195659,0 & 7505229,9 & 0,72 & 0,81 & 0,94 & 1,18 & $-0,26$ & $-0,36$ & 3.22 & 2.42 \\
\hline $\mathrm{H}_{\boldsymbol{v}-i}$ & 195880,4 & 7505278,8 & 1,83 & 0,34 & 1,20 & 1,18 & $-1,48$ & 0,84 & 4.72 & 3.22 \\
\hline
\end{tabular}
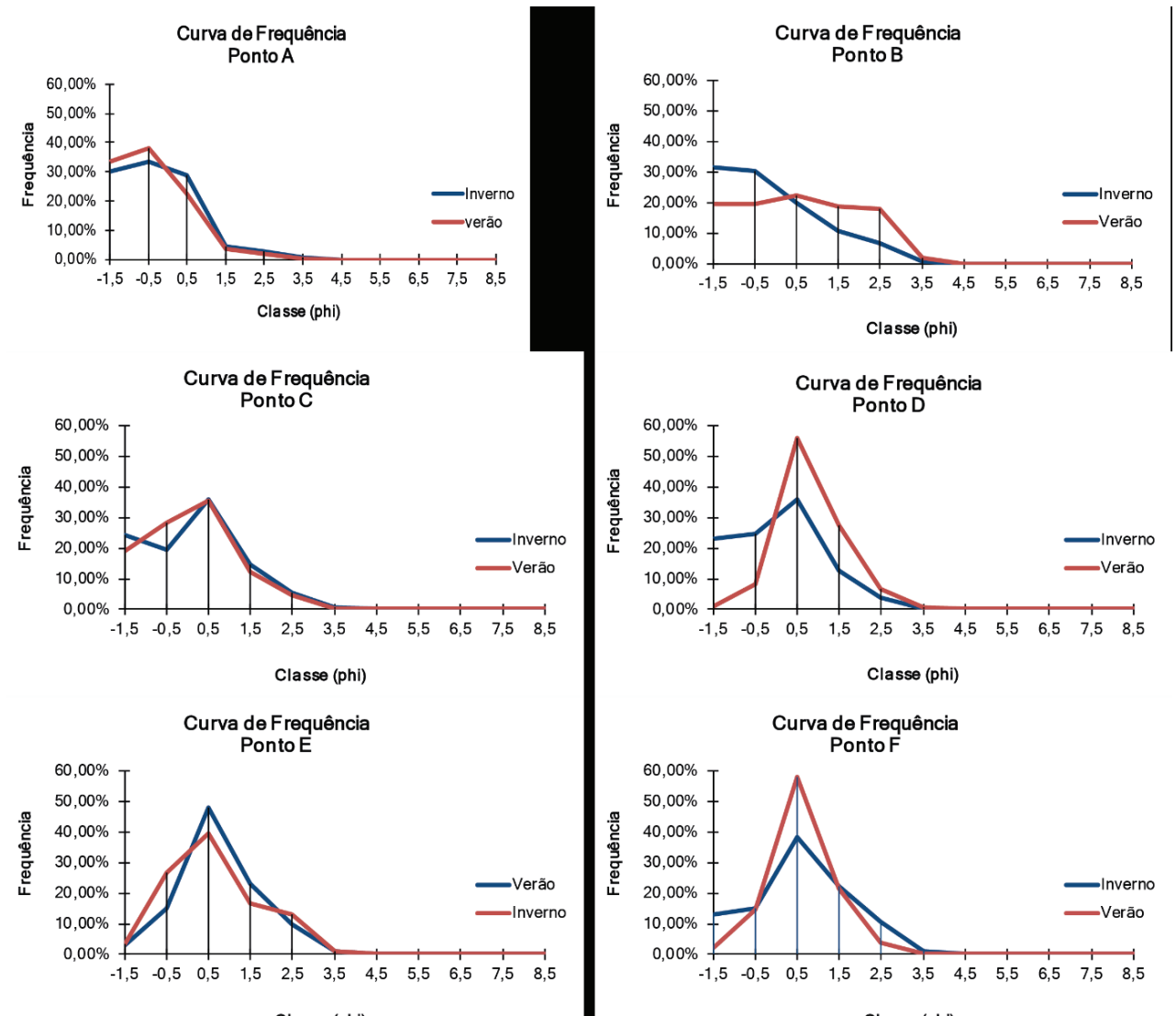

Curva de Frequência
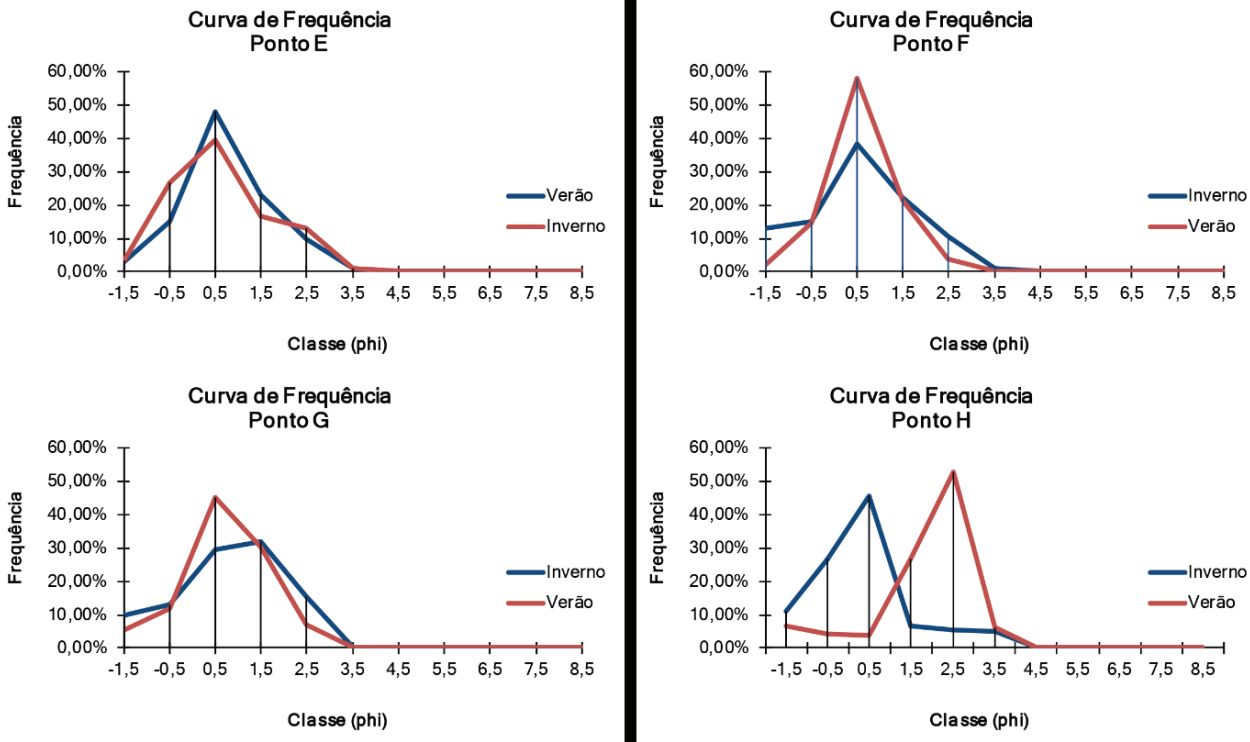

Figura 2 - Curvas de dispersão granulométrica em Phi para os 8 (oito) pontos de coleta. As curvas em vermelho representam as distribuições para amostras coletadas durante campanhas de verão de 2010 e as azuis representam as distribuições de amostras coletadas durante campanhas de inverno de 2009. 
4.2 Clima de Ondas, Definição de Parâmetros Hidrodinâmicos

Resultados do clima de ondas da região são apresentados de forma sintética na figura 3, conforme série histórica de 36 anos obtida através do BNDO - Marinha do Brasil. Estatisticamente, representam a moda das alturas de ondas $\left(H_{0}\right)$, períodos $(T)$ e direções de ondas em águas profundas. Resultados sugerem que o clima de onda é caracterizado por ondulações do quadrante nordeste, com menor frequência de leste-sudeste. Os valores obtidos para $H_{0}$ e $T$ foram, respectivamente, $1,5 \mathrm{~m} / 5 \mathrm{~s}$ e $1,0 \mathrm{~m} / 5$ para períodos de inverno e verão.
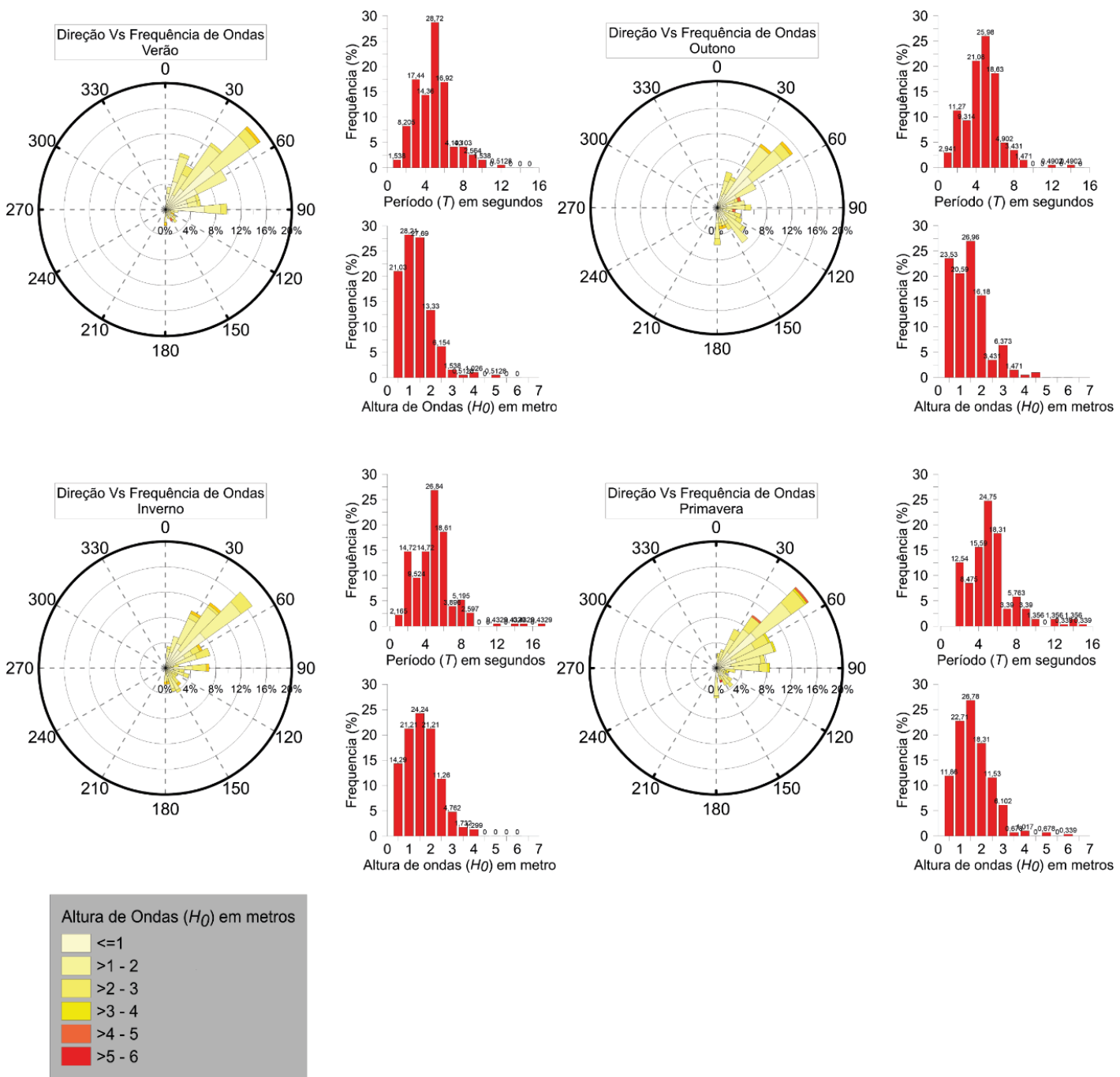

Figura 3 - Roseta de direções de ondas e associadas aos histogramas de alturas $\left(H_{0}\right)$ e periodos $(T)$ de ondas em água profundas, para as quatro estações climáticas. Estatística da série histórica, compreendida entre os anos de 1963 a 1999. Base de dados fornecida pelo Banco de Dados Oceanográficos - BNDO da Marinha do Brasil.

Após a definição das alturas, períodos e direções de ondas predominantes, foi possível, através da equação proposta por Weishar \& Byrne (1978), determinar $H b$, bem como a respectiva $h b$. Os resultados foram estimados, respectivamente, em 1,38 m e 1,77 m para período de inverno e $1,17 \mathrm{~m} \mathrm{e} 1,50 \mathrm{~m}$ para período de verão.

\subsection{Estimativa de Taxa de Transporte de Sedimentos}

Na tabela 2 são apresentados resultados, ponto a ponto, para estimativas das taxas de transporte de sedimentos induzidas por ondas em vazões sólidas, $\boldsymbol{Q}_{s}\left(\mathrm{~m} 3 . \mathrm{s}^{-1}\right)$ e vazões sólidas residuais, $\boldsymbol{Q}_{s \boldsymbol{r}}\left(\mathrm{m}^{3}\right.$.ano $\left.{ }^{-1}\right)$ (CERC, 1984). As taxas foram calculadas para os períodos de inverno e verão, considerando as variações 
de $H s, T$ e o $\mathrm{D}_{50}$, bem como a frequência nas direções de ondas significativas que atingem o litoral durante as respectivas estações. Convencionalmente, os resultados apresentam sinais positivos ou negativos em decorrência do referencial adotado. Sendo assim, valores positivos representam transporte residual no sentido sudeste e valores negativos associados ao transporte residual no sentido nordeste.

Tabela 2: Resultados das taxas de transporte de sedimento induzido por ondas (CERC, 1984) em m3.s-1 e taxas residuais anuais em m3.ano-1. Valores positivos representam transporte residual no sentido sudeste e valores negativos representam transporte residual no sentido nordeste.

\begin{tabular}{|c|c|c|c|c|c|c|}
\hline $\begin{array}{l}\text { Pontos de } \\
\text { Amostras }\end{array}$ & $\begin{array}{c}\mathbf{D}_{50} \\
(\mathbf{m m})\end{array}$ & $\begin{array}{l}H_{b} \\
(\mathbf{m})\end{array}$ & $\begin{array}{c}T \\
(\mathbf{s})\end{array}$ & $\begin{array}{c}\text { Direção de } \\
\text { ondas/frequência }\end{array}$ & $\underset{\left(\mathrm{m}^{3} \cdot \mathrm{s}^{-1}\right)}{Q_{s}}$ & $\underset{\substack{Q_{s r} \\
\text { residual } \\
\left(\mathbf{m}^{3} \cdot \text { ano }^{-1}\right)}}{ }$ \\
\hline \multirow{2}{*}{$\mathbf{A}_{v}$} & \multirow{2}{*}{1,38} & \multirow{2}{*}{1,38} & \multirow{4}{*}{5} & $\mathrm{NE}(18 \%)$ & $+0,00373$ & \multirow{4}{*}{$+75.369,7$} \\
\hline & & & & $\operatorname{SSE}(3 \%)$ & $-0,00481$ & \\
\hline \multirow{2}{*}{$\mathbf{A}_{i}$} & \multirow{2}{*}{0,94} & \multirow{2}{*}{1,17} & & $\mathrm{NE}(17 \%)$ & $+0,01827$ & \\
\hline & & & & SSE (5\%) & $-0,02486$ & \\
\hline \multirow{2}{*}{$\mathbf{B}_{v}$} & \multirow{2}{*}{0,69} & \multirow{2}{*}{1,38} & \multirow{4}{*}{5} & NE $(18 \%)$ & $+0,02144$ & \multirow{4}{*}{$+134.170,8$} \\
\hline & & & & $\operatorname{SSE}(3 \%)$ & $-0,02831$ & \\
\hline \multirow{2}{*}{$\mathbf{B}_{i}$} & \multirow{2}{*}{1,12} & \multirow{2}{*}{1,17} & & NE $(17 \%)$ & $+0,01199$ & \\
\hline & & & & SSE (5\%) & $-0,01589$ & \\
\hline \multirow{2}{*}{$\mathbf{C}_{v}$} & \multirow{2}{*}{0,95} & \multirow{2}{*}{1,38} & \multirow{4}{*}{5} & NE $(18 \%)$ & $+0,01002$ & \multirow{4}{*}{$+106.807,6$} \\
\hline & & & & SSE $(3 \%)$ & $-0,01323$ & \\
\hline \multirow{2}{*}{$\mathbf{C}_{i}$} & \multirow{2}{*}{0,94} & \multirow{2}{*}{1,17} & & $\mathrm{NE}(17 \%)$ & $+0,01909$ & \\
\hline & & & & $\operatorname{SSE}(5 \%)$ & $-0,02530$ & \\
\hline \multirow{2}{*}{$\mathbf{D}_{v}$} & \multirow{2}{*}{0,56} & \multirow{2}{*}{1,38} & \multirow{4}{*}{5} & $\mathrm{NE}(18 \%)$ & $+0,029732$ & \multirow{4}{*}{$+\mathbf{1 8 6 . 0 5 5 , 1}$} \\
\hline & & & & SSE (3\%) & $-0,039253$ & \\
\hline & & & & NE $(17 \%)$ & $+0,016635$ & \\
\hline $\mathbf{D}_{i}$ & 1,00 & 1,17 & & $\operatorname{SSE}(5 \%)$ & $-0,022050$ & \\
\hline & 059 & & & NE $(18 \%)$ & $+0,027488$ & \\
\hline $\mathbf{E}_{v}$ & 0,59 & 1,38 & 5 & $\operatorname{SSE}(3 \%)$ & $-0,03629$ & \\
\hline & & & 5 & $\mathrm{NE}(17 \%)$ & $+0,039978$ & +243.805,5 \\
\hline $\mathbf{E}_{i}$ & 0,64 & 1,17 & & SSE $(5 \%)$ & $-0,052990$ & \\
\hline & 0,77 & & & NE $(18 \%)$ & $+0,017588$ & \\
\hline $\mathbf{F}_{v}$ & & 1,38 & & SSE (3\%) & $-0,023220$ & \\
\hline & 0,68 & & 5 & NE $(17 \%)$ & $+0,036381$ & +196.877 \\
\hline $\mathbf{F}_{i}$ & & 1,17 & & $\operatorname{SSE}(5 \%)$ & $-0,048221$ & \\
\hline & 0,60 & & & $\mathrm{NE}(18 \%)$ & $+0,026755$ & \\
\hline $\mathbf{G}_{v}$ & & 1,38 & 5 & SSE (3\%) & $-0,035323$ & +2754801 \\
\hline & 0,57 & & 5 & NE $(17 \%)$ & $+0,048005$ & $+2 / 5.489,1$ \\
\hline $\mathbf{G}_{i}$ & & 1,11 & & SSE $(5 \%)$ & $-0,063629$ & \\
\hline $\mathbf{H}_{-}$ & 028 & 138 & & NE $(18 \%)$ & $+0,059724$ & \\
\hline $\mathbf{H}_{v}$ & 0,28 & 1,38 & 5 & $\operatorname{SSE}(3 \%)$ & $-0,078849$ & \\
\hline $\mathrm{H}_{i}$ & 078 & 117 & 5 & $\mathrm{NE}(17 \%)$ & $+0,055574$ & $+514.18 /, 7$ \\
\hline $\mathbf{H}_{i}$ & 0,78 & 1,17 & & SSE (5\%) & $-0,073662$ & \\
\hline
\end{tabular}

As taxas de transporte pontuais, expressas em $\mathrm{m}^{3} . \mathrm{s}^{-1}$, apresentam valores superiores para ondas oriundas de SSE. No entanto, as taxas residuais médias anuais, apresentadas $\mathrm{em} \mathrm{m}^{3}$.ano ${ }^{-1}$, apontam a direção preferencial de transporte longitudinal de NE para SSE. Indicando o aumento na capacidade de transporte de $\mathbf{A}$ para $\mathbf{H}$, estimado em $7,5 \times 10^{4} \mathrm{~m}^{3} \cdot \mathrm{ano}^{-1}$ e $5,1 \times 10^{5} \mathrm{~m}^{3} \cdot \mathrm{ano}^{-1}$, respectivamente.

\section{Discussão}

Os resultados obtidos para o diâmetro mediano do grão $\left(\mathrm{D}_{50}\right)$ nos oito $(8)$ pontos de coleta sugerem a prevalência de sedimentos grossos a muito grossos e moderadamente selecionados, exceto no ponto $\mathbf{H}$, onde os resultados indicaram a presença de areias médias para período de verão. Conforme Bird (2008), a ocorrência de alta concentração de sedimentos grossos em praias, 
relaciona-se a três aspectos sedimentológicos distintos. O primeiro, trata-se da remoção dos sedimentos mais finos pela ação das correntes de retorno "rip current" e de deriva litorânea "longshore current". O segundo, relaciona-se ao aporte de sedimentos provenientes de desembocaduras fluviais e o último, associa-se a eventos extremos de tempestade. Castro et al. (2011) identificaram também uma tendência a diminuição na granulometria do ponto A para o ponto $\mathbf{H}$, isto é, de sul para norte. Essa observação é registrada, tanto nas amostras coletadas durante período de inverno quanto no verão. Possivelmente, este processo de "granodecrecência", atribui-se a entrada de "Sistemas Frontais" identificados pela ação de ondas longínquas (swell) que atingem o litoral do estado do Rio de Janeiro durante o inverno (Parise et al., 2009), resultando assim, numa inversão da deriva e consequentemente na diminuição do padrão granulométrico local.

Os valores estabelecidos de curtose são distintos para períodos de inverno e verão. Também ocorrem variações em função da localização das amostras coletadas. Formas platicúrticas e mesocúrticas são verificadas nas curvas de distribuição granulométrica para sedimentos coletados durante o inverno, enquanto formas leptocúrticas durante o verão. Nos pontos localizados mais a sul da área de estudo (A, B e C) são observadas também curvas com maior dispersão granulométrica.

Fisicamente, estes resultados relacionam-se a dois importantes aspectos observados na aérea de estudo. $\mathrm{O}$ primeiro refere-se à preservação das propriedades dos sedimentos, desde da área fonte até a sua deposição (Folk \& Ward, 1957). Considerando a proximidade da desembocadura do rio São João, as amostras coletadas mais a sul da área de estudo apresentariam maior dispersão das classes granulométricas e consequentemente, curvas de distribuição mais atenuadas, com formas platicúrticas. O segundo aspecto, diz respeito a alternância das características físicas dos sedimentos em decorrência da ciclicidade de eventos meteorológicos, modificando o estado de mar e as direções de derivas litorâneas.

Valores de assimetria positiva foram observados nas amostras coletadas entre os pontos A e E. Segundo Friedman (1967), areias de praia apresentam, tipicamente, valores de assimetria mais negativa, enquanto areias fluviais apresentariam valores de assimetria mais positiva. Entretanto, as diferenças texturais também são atribuídas a disponibilidade de sedimentos das áreas fontes. De acordo com Duane (1964), valores de assimetria positiva podem ocorrer em virtude de agentes externos ao ambiente praial. Desta forma, os valores de assimetria positivas estariam relacionados diretamente a desembocadura do São João, a sul da área de estudo.

Os dados referentes ao clima de ondas da região foram divididos em espectros de ondas para cada estação do ano, conforme direção e frequência. Verificou-se que os trens de ondas provenientes do quadrante nordeste representam aproximadamente $40 \%$ do total da frequência para todas as estações do ano. As ondulações provenientes de nordeste com ângulo de $50^{\circ}$ representam $18 \%$ durante os meses de primavera e verão, $17 \%$ durante os meses de inverno e 15\% para a estação de outono. Em contraponto, as ondas provenientes de sudeste, representam de $3 \%$ a $10 \%$ do total de ondas incidentes. Durante os meses de verão as ondas provenientes do ângulo $150^{\circ}$ correspondem ao quadrante sul-sudeste com $3 \%$ da frequência total, enquanto no inverno este valor se aproxima de 5\%. Os resultados obtidos nesse trabalho aproximam-se das informações referentes aos trabalhos desenvolvidos por Silva (2009) e Castro (2018).

Apesar da pequena discrepância em relação ao trabalho desenvolvido por Silva (2009) na Enseada dos Anjos - Arraial do Cabo / RJ, os resultados obtidos aqui vão ao encontro de trabalhos desenvolvidos anteriormente na bacia de Campos, que definiram o clima de ondas como predominante de tempo bom, com ondulações variando no quadrante nordeste e ocorrência de marulhos provenientes de Anticiclones do Atlântico Sul (AAS) associado com sistemas frontais e frentes frias polares, com direção leste-sudeste (Cassar e Neves, 1993; Muehe, 1998; Pinho, 2003; Rangel, 2005; Savi, 2007; Parente et al., 2014; Castro, 2018).

A formulação proposta por Weishar \& Byrne (1978) forneceu estimativa razoável em relação às alturas das ondas na arrebentação através do período e altura das ondas em águas profundas. No entanto, os efeitos da refração ou dissipação pela friç̧ão não foram incorporados neste trabalho. Para o cálculo das estimativas de transportes de sedimentos longitudinais, utilizou-se a equação do CERC (USACE, 1984). Essa formulação matemática, relaciona a potência transmitida paralelamente à praia a capacidade de transporte de sedimento (USACE, 2002a). Os dados para formulação dessa equação foram obtidos empiricamente em praias dos Estados Unidos, México e Japão, constituídas por 
grãos de quartzo com o $\mathrm{D}_{50}$ variando de 0,20 a 1,0 mm (USACE, 2002b).

As taxas residuais $(Q s r)$ obtidas para as praias das Tartarugas e Abricó apresentaram grande variação em virtude da distribuição granulométrica. Os valores de $75.369,7 \mathrm{~m}^{3}$.ano ${ }^{-1}$ no ponto A e $514.187,7 \mathrm{~m}^{3}$. ano ${ }^{-1}$ no ponto $\mathbf{H}$, são estimativas da capacidade de transporte de sedimentos em virtude das propriedades físicas dos sedimentos e a energia disponibilizada pelas ondas na região. Ainda que haja um histórico de recuo da linha de praia, incluindo a destruição de infraestruturas urbanísticas, (Castro et. al., 2011; Muehe et. al., 2011) estes resultados não podem ser relacionados diretamente com variações nas taxas de recuo em diferentes pontos da mesma. Isto se deve, além da própria fisiografia da praia, à intervenção antrópica em diversos pontos da costa, como em corpos hídricos que desaguam neste trecho do litoral.

A taxa residual média $(Q \mathrm{srm})$ foi estabelecida em 216.595,3 $\mathrm{m}^{3}$.ano ${ }^{-1}$ no sentido sul, apesar das vazões sólidas $(Q s)$, expressas $\mathrm{em} \mathrm{m}^{3} \cdot \mathrm{s}^{-1}$, apresentarem valores superiores para ondas oriundas de SSE. Tal resultado, deve-se ao fato das frequências de ondas de NE serem até 6 vezes maior que de SSE (figura 3), sendo assim capazes de remobilizar um volume superior de sedimentos ao longo do tempo.

O resultado obtido da taxa residual média ficou bem abaixo das taxas estimadas para outros segmentos do litoral Brasileiro. Lima et al. (2002), estimou as taxas de $2.900 .000 \mathrm{~m}^{3}$.ano ${ }^{-1}$ para a praia do Hermenegildo e $2.600 .000 \mathrm{~m}^{3}$. ano $^{-1}$ entre as praias do Cassino e Solidão, ambas no Rio Grande do Sul. Martins et al. (2004) estimou a taxa de transporte próximo à desembocadura sul do complexo estuário de Paranaguá em 1.500.000 $\mathrm{de}^{3}$.ano ${ }^{-1}$. Alfredini (1999) calculou valores da ordem de 2.200.000 de $\mathrm{m}^{3}$. ano $^{-1}$ na praia de Brejatuba em Guaratuba, Paraná. No litoral norte fluminense, Cassar $\&$ Neves (1993) estimaram taxas de $2.500 .000 \mathrm{~m}^{3}$.ano ${ }^{-1}$ a $3.400 .000 \mathrm{~m}^{3}$. ano $^{-1}$ com sentido para sul nas praias de Itabapoana, Usina e Guaxindiba, região ao norte da desembocadura do Paraíba do Sul. A sul da desembocadura do Paraíba do Sul, na praia de Atafona, região que sofre intenso processo erosivo, a taxa de transporte de sedimentos foi estimada em $1.500 .000 \mathrm{~m}^{3} . \mathrm{ano}^{-1}$.

O transporte de sedimentos nas zonas costeiras é um tema ainda a ser amplamente estudado. O mesmo depende de uma série de parâmetros e variáveis físicas, geológicas e oceanográficas, muitas destas de difícil aferição em campo e laboratório. Dessa forma, muitos dos modelos matemáticos, físicos e empíricos, tentam representar, de forma simplificada, os eventos que ocorrem naturalmente no meio ambiente (Van Rijn, 1993). Conforme Schoonees \& Theron (1994) as discrepâncias nos resultados obtidos para as taxas de transporte de sedimento litorâneo são comuns e usuais, devendo-se às condições ambientais, à natureza dos fluidos e aos métodos empregados.

\section{Conclusão}

De acordo com os objetivos propostos neste artigo, chegou-se as seguintes conclusões em relação as estimativas de taxas de transporte de sedimentos induzidos por ondas nas praias das Tartarugas e Abricó, segmento norte do arco praial da Baia Formosa, Rio das Ostras / Estado do Rio de Janeiro:

- Verificou-se uma tendência granulométrica granodecrescente em direção a norte e diminuição do grau de assimetria $(S k)$ no mesmo sentido. Observou-se que nas proximidades do Rio São João, a distribuição granulométrica é mais homogênea, sugerindo que esse sistema fluvial é a principal fonte de sedimentos para a plataforma continental interna;

- O clima de ondas da região é predominantemente de nordeste com alturas em águas profundas $\left(H_{0}\right)$ entre 1,0 e 2,0 m e período $(T)$ em torno de $5 \mathrm{~s}$. Ondas provenientes de sul-sudeste apresentam de $3 \%$ e $5 \%$ das frequências nos meses de verão e inverno, respectivamente. No entanto, são responsáveis aproximadamente por $30 \%$ do volume total de sedimentos transportados pela deriva litorânea;

- Verificou-se que o rumo do transporte longitudinal de sedimentos litorâneo na zona de arrebentação, frontal às praias das Tartarugas e Abricó apresenta sentido predominantemente para sul. A magnitude calculada através do método utilizado para estimativas de transporte de sedimento foi avaliada em $75.369,7 \mathrm{~m}^{3}$.ano ${ }^{-1}$ a $514.187,7 \mathrm{~m}^{3}$. ano $^{-1}$, com média (Qsrm) de 216.595,3 $\mathrm{m}^{3} /$ ano.

Portanto, os valores apresentados em relação às estimativas de taxas de transporte de sedimentos induzidos por ondas mostraram-se relativamente baixos, comparado as taxas calculadas para o litoral norte do Estado do Rio de Janeiro. Denotando assim, baixo 
índice de mobilidade de sedimentos, apesar do intenso processo de erosão em curso neste trecho de litoral. Sendo assim, considera-se os valores obtidos apenas razoáveis à implementação de possíveis obras de engenharia costeira, uma vez que essa ordem de grandeza para o transporte de sedimentos deverá gerar respostas lentas da linha de praia.

\section{Agradecimentos}

Ao Conselho Nacional de Pesquisa - CNPq pela cessão da bolsa de doutorado e a Fundação Carlos Chagas Filho de Amparo à Pesquisa do Estado do Rio de Janeiro - FAPERJ pelo suporte financeiro aos projetos do Laboratório de Geologia Costeira, Sedimentologia e Meio Ambiente - LAGECOST, do Museu Nacional / UFRJ.

\section{Referências Bibliográficas}

ALFREDINI, P. The longshore transport evaluation used as a tool for the brazilian coastline littoral processes knowledge. In: Anais do VII Congresso Brasileiro da Associação Brasileira de Estudos do Quaternário (ABEQUA), 7., 13 p, 1999.

ALVES, E. C. \& PONZI, V.R.A. Características morfológicosedimentares da plataforma continental e talude superior da margem continental sudeste do Brasil. In: XXXIII CONGRESSO BRASILEIRO DE GEOLOGIA, 32., 1984, Rio de Janeiro-RJ. p.1629-1642, 1984.

BILARD, J.A. \& INMAN, D.L. An energetics bedload model for plane sloping beach: local transport. Journal of Geophysical Research, v. 86, (C3), p. 2035-2043, 1981.

BAYRAM, A.; LARSON, M.; MILLER, H.C.; KRAUS, N.C. Crossshore distribution of longshore sediment transport: comparison between predictive formulas and field measurements. Coastal Engineering, v. 44, p.79-99, 2001.

BIRD, E.C.F. Beach Management. New York, John-Wiley and Sons, 1999, 292 p.

BIRD, E.C.F. Coastal geomorphology: an introduction. $2^{\mathrm{a}}$ ed. _ New York: John Wiley \& Sons, 2008. 436p.

BIJKER, E.W. Some considerations about scales for coastal models with movable beds. Delft Hydraulics Laboratory, Netherlands, n. 50, 1967.

CARVAlho, V. C. \& RizZO, H. G. A. Zona Costeira Brasileira: subsídios para uma avaliação ambiental. Ministério do Meio Ambiente e da Amazônia Legal. Brasileira, MMA, 1994.

CASSAR, J.C.M. \& C.F. NEVES (1993). Aplicação das rosas de transporte litorâneo à costa norte fluminense. Revista Brasileira de Engenharia (RBE), Caderno de Recursos Hídricos. v. 11, (1): p. 81-106. Associação Brasileira de Recursos Hídricos ABRH, São Paulo, SP.

CASTRO, J.W.A; FERNANDES; D., DIAS, F. Monitoramento do Processo de Erosão Costeira na Praia das Tartarugas, Rio das Ostras - Estado do Rio de Janeiro / Brasil: Aplicação de Metodologia Quantitativa, Revista da Gestão Costeira Integrada, p. 356-368, 2011.

CASTRO, J.W.A; SUGUIO, K; SEOANE, J.C.S; CUNHA, A.M; DIAS, F.F. Sea-level fluctuations and coastal evolution in the state of Rio de Janeiro, southeastern Brazil. Annals of the Brazilian Academy of Sciences. v. 86 (2), p. 671 - 683, 2014.

CASTRO, J. W. A. (2018). The Morphodynamics Behaviour of a Cross-Shore Sandbar in a Microtidal Environment, Anjos Cove, Arraial do Cabo, Rio de Janeiro - Brazil. In: Botero, Camilo M., Cervantes, Omar D., Finkl, Charles W. (Org.). Beach Management Tools - Concepts, Methodologies and Case Studies. 1ed.Berlin - Alemanha: Springer (Coastal Research Library), v. 24, p. $319-329$

CERC. Shore Protection Manual. Vicksburg, Mississippi: U.S Army Coastal Engineering Research Center, Corps of Engineers. Vol. I, II, 1984.

DEAN, R. G. Beach nourishment: theory and practice. Prentice-Hall, p. 399, 2000.

DEAN, R. \& DARLYMPLE, R. Coastal Processes with Engineering Applications, Cambridge University, 2002. 475 p.

DUANE, D.B. Significance of skewness in recent sediments, Western Pamlico Sound, North Carolina. Journal of Sedimentary Petrology, v. 34: 864-874, 1964.

FERNANDEZ, G. B. \& MUEHE, D. Cobertura sedimentar recente e batimetria da plataforma continental interna entre Macaé e o Cabo Búzios, RJ. In: VI Simpósio Nacional de Geografia Física Aplicada, 6.,1995, Anais. v. 1, p. 196-203. 1995.

FERNANDEZ, G. B. \& MUEHE, D. A influência de sedimentos fluviais na morfologia da praia e antepraia no embaiamento Rio das Ostras - Cabo Búzios - (RJ). Geosul. v. 14, n. 27, p. 200-203, 1998.

FOLK, R. \& WARD, W. Brazos river bar. A study in the significance of grain size parameters. Journal of. Sedmentary 
Petrology. v. 27 (1), p. 3-26, 1957.

FRIEDMAN, G. M., On sorting, sorting coefficients, and the lognormality of the grain size distribution of sandstones. Journal of. Geology., 70: 737-756, 1962b.

FRIEDMAN, G. M. Dynamic processes and statistical parameters compared for size frequency distribution of beach and river sands. Journal of Sedimentary Petrology, v. 37, p. 327-354, 1967.

HAAS, K.A. \& HAYNES, D.M. Process based modeling of total longshore sediment transport. Journal of Coastal Research, v. 20 (3), p. 853-861, 2004.

KOMAR, P.D. \& INMAN, D.L. Longshore sand transport on beaches. Journal of Geophysical Research, v. 75, p. 5514-5527, 1970.

KOMAR, P.D. Beach processes and sedimentation. New Jersey: Prentice Hall, 1976, p. 429

KOMAR, P., 1988. Environmental controls on littoral sand transport. Proceedings 21st Coastal Engineering Conference (ASCE), p. 1238-1252.

LIMA, S.F., ALMEIDA, L.E.S.B \& TOLDO, JR.E.E. Estimativa da capacidade do transporte longitudinal de sedimentos a partir de dados de ondas para costa do Rio Grande do Sul. Pesquisas em Geociências, v. 28 (2): p. 99-107, 2002.

LONGUET-HINGINS \& STEWART. Radiation stresses in water waves; a physical discussion, with applications. Deep-Sea Research, Pergamon, Vol. II, p. 529 - 562.1964

MARTINS, G., MARONE, E, Ângulo, R.J., Noernberg, M.A., Quadros, C.J.L. Dinâmica da zona rasa de shoaling e o transporte de sedimentos na desembocadura sul do Complexo Estuarino de Paranaguá-PR. Boletim Paranaense de Geociências, n. 54, p. 51-64, 2004.

MUEHE D. \& VALENTINI, E. O Litoral do Rio de Janeiro: uma caracterização físico-ambiental. Rio de Janeiro, FEMAR, 1998,123 p.

MUEHE, D. (1998) O litoral brasileiro e sua compartimentação. In Cunha SB \& Guerra AJT (orgs) Geomorfologia do Brasil. Rio de janeiro, Bertrand Brasil, p 273-349.

MUEHE, D., FERNANDEZ, G. B., BULHÕES, E. M. R., AZEVEDO, I. F. Avaliação da vulnerabilidade física da orla costeira em nível local, tomando como exemplo o arco praial entre Rio das Ostras e o Cabo Búzios/RJ. Revista Brasileira de Geomorfologia, v.12, n.2, p.45-58, 2011

OLIVEIRA, J.F \& MUEHE, D. Identificação de áreas de sedimentos compatíveis na plataforma continental

interna para recuperação de praias entre as cidades de Niterói e Macaé - Rio de Janeiro, Brasil. Revista de Gestão Costeira Integrada. v. 13(1): p. 89-99, 2013.

PARENTE, C.E.; NOGUEIRA, I.C.M.; MARTINS, R.P. e Ribeiro, E.O. Climatologia de Ondas. In: Martins, R.P. \& Matheson, G.S.G (editores). Caracterização Ambiental Regional da Bacia de Campos, Atlântico Sudoeste: Meteorologia e Oceanografia. Rio de Janeiro: Elsevier, in press (Série Habitats). 2014.

PARISE C.K.; CALLIARI L.J.; Krusche N. Extreme storm surges in the South of Brazil: Atmospheric conditions and shore erosion. Brazilian Journal of Oceanography, v. 57 (3): p. 175-188, 2009.

PINHO, U. F. Caracterização dos Estados de Mar na Bacia de Campos [Rio de Janeiro]. Dissertação de Mestrado - Universidade Federal do Rio de Janeiro, COPPE/UFRJ, Engenharia Oceânica, 2003, 123 p.

SAVI, D.C. Erosão e acreção costeira na enseada dos Anjos, Arraial do Cabo, RJ. Revista Brasileira de Geofísica, v. 25, suplemento 1, p 91-100, 2007.

SCHOONEES, J. S.; THERON, A. K. (1994). Accuracy and applicability of the SPM longshore transport. Coastal Engineering 1994. ASCE, Kobe Japan, v. 3, 2595-2609.

SHORT, A.D. \& HOGAN, C.L., A.D. Rip Currents and Beach Hazards: Their Impact on Public Safety and Implications for Coastal Management. Journal of Coastal Research. v.12, COASTAL HAZARDS: PERCEPTION, SUSCEPTIBILITY AND MITIGATIO, p. 197-209, 1994.

SILVA, C.G. et al. Ambientes de sedimentação costeira e processos morfodinâmicos atuantes na linha de costa. In Baptista Neto, J.A, et al (org): Introdução a Geologia marinha, Interciência, Rio de Janeiro, p. 175-218, 2004.

SILVA AC. Dinâmica Batimétrica e Sedimentológica da Região do Cabo Frio - Estado do Rio de Janeiro. Unpublished $\mathrm{PhD}$ Thesis, Universidade Federal do Rio de Janeiro, Rio de Janeiro, 2009, 157 p.

SOUZA, C.R.G. (2009). A Erosão nas Praias do Estado de São Paulo: Causas, Consequências, Indicadores de Monitoramento e Risco. In: V.L.R. Bononi \& N.A. SantosJúnior (orgs.). Memórias do Conselho Científico da Secretaria do Meio Ambiente: A Síntese de Um Ano de Conhecimento Científico Acumulado. Secretaria de Meio Ambiente do Estado de São Paulo, São Paulo, SP, Brasil. 
Transporte de Sedimentos Induzido por Ondas no Segmento Norte da Enseada da Baia Formosa, Rio das Ostras

USACE (US ARMY CORPS OF ENGINEERS), Water Wave Mechanics. In: USACE. Coastal Engineering Manual Chapter 1, Part II. Vicksburg, Mississippi: EM 1110-2-1100, 2002a. 121p.

USACE (US ARMY CORPS OF ENGINEERS), Longshore Sediment Transport. In: USACE. Coastal Engineering Manual

- Chapter 2, Part III. Vicksburg, Mississippi: EM 1110-2-1100, 2002b. 113p.

VAN RIJN, L.C. Sediment transport: Part I; Part II; Part III. Journal of Hydraulic Division, 1984, 110 (10), 1431-1456; 110 (11) $1613-1641 ; 110$ (12) 1733-1754.
VAN RIJN, L.C. Principles of sediment transport in rivers, estuaries and coastal seas. Amsterdam, Holanda: Aqua Publications, 1993.

VAN RIJN, L.C. Principles of Coastal Morphology. Amsterdam, Holanda: Aqua Publications, 1998.

WEISHAR, L.L. \& BYNE, R.J. Field study of breaking wave characteristics. Proceedings of the 16th Coastal Engineering Conference. ASCE, New York, 487-506 p, 1978

WRIGHT, L.D., SHORT, A.D., 1984. Morphodynamic Variability of Surf Zones and Beaches: Sybthesis* Marine Geology, v. 56, p. 93-118, 1978. 